\title{
Computation of the Field in an Axial Gap, Trapped-Flux Type Superconducting Electric Machine
}

\author{
Zejun Shen, Mark D. Ainslie, Archie M. Campbell, and David A. Cardwell
}

\begin{abstract}
The Bulk Superconductivity Group at the University of Cambridge is currently investigating the use of high temperature superconductors in wire and bulk form to increase the electrical and magnetic loading of an axial gap, trapped flux-type superconducting electric machine. The use of superconducting materials in electric machines can lead to increases in efficiency, as well as power density, which results in reductions in both the size and weight of the machine. In this paper, the authors present a method to compute the field in such an electric machine generated by an array of fully magnetized bulk superconductors. Analytical expressions are derived for the field that would exist in the coil region of the motor, which will act as a powerful tool for carrying out parametric analysis of the motor's design and performance.
\end{abstract}

Index Terms-Electric machines, high-temperature superconductors, permanent magnet machines, superconducting magnets, superconducting materials.

\section{INTRODUCTION}

$\mathbf{S}$ UPERCONDUCTING electric machines are an important application of superconducting materials in both bulk and wire forms. Bulk high temperature superconductors, in particular, are capable of trapping magnetic fields greater than $17 \mathrm{~T}$ below $30 \mathrm{~K}$ [1], [2], as well as up to $3 \mathrm{~T}$ at the technologically important temperature of $77 \mathrm{~K}$ [3]. Such superconductors can replace permanent magnets in the rotor of a traditional machine, resulting potentially in electric machines that are smaller and lighter than conventional designs [4]-[7]. Detailed analysis of the magnetic field of the superconductors is key to the design and optimization of the machine, and can assist in determining the performance of such machines in terms of torque, and hence, power, efficiency, and so on. A number of different methods have been used to calculate the field generated by bulk superconductors, including analytical methods [8]-[11] and numerical methods [12]-[14].

Manuscript received August 4, 2014; accepted October 21, 2014. Date of publication November 4, 2014; date of current version January 19, 2015. This work was supported in part by a Royal Academy of Engineering Research Fellowship and by the China Scholarship Council.

Z. Shen is with the Department of Electrical Engineering, Tsinghua University, Beijing 100084, China. He is also with the Bulk Superconductivity Group, University of Cambridge CB2 1PZ, U.K. (e-mail: szj10@mails. tsinghua.edu.cn).

M. D. Ainslie, A. M. Campbell, and D. A. Cardwell are with the Bulk Superconductivity Group, Department of Engineering, University of Cambridge, Cambridge CB2 1PZ, U.K. (e-mail: mark.ainslie@eng.cam.ac.uk).

Color versions of one or more of the figures in this paper are available online at http://ieeexplore.ieee.org.

Digital Object Identifier 10.1109/TASC.2014.2366972

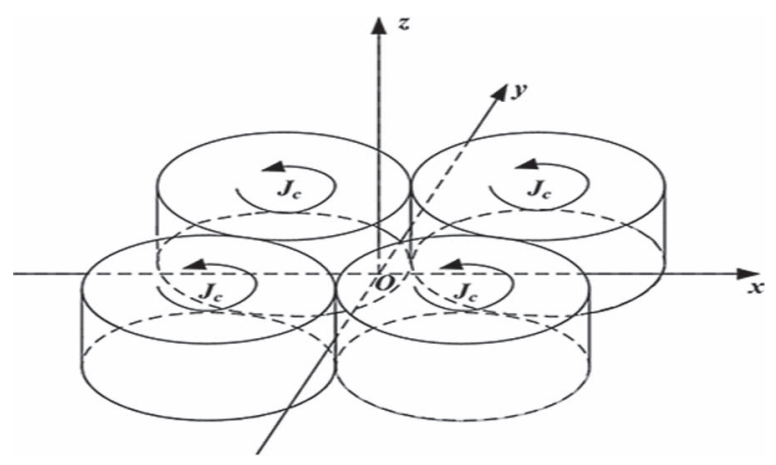

Fig. 1. The model of the four-bulk superconductor array under analysis.

Analytical methods are easier, faster and more accurate compared to numerical methods, such as the Finite Element Method [15], but are limited to specific geometries and simplified assumptions. In this paper, different analytical methods-the Biot-Savart Method, the Fourier Transform Method (FT), and Fast Fourier Transform Method (FFT) - are used to calculate the magnetic field in an axial gap, trapped-flux type superconducting electric machine. The results of these methods are compared using a number of metrics to determine their efficacy.

\section{BulK Superconductor Model}

The model of four fully magnetized bulk superconductors used for this analysis is shown in Fig. 1. This is representative of an array of bulk superconductors used as a magnetic field pole in an axial gap, trapped-flux type superconducting electric machine. The distribution and dimensions of the superconductors are based on [9]. The diameter of the superconductors is $d=10 \mathrm{~mm}$, and each has a height of $h=10 \mathrm{~mm}$. The $x-y$ plane $(z=0)$ corresponds to the midpoint of the height of the superconductors. The centre of the superconductors are located at $( \pm 5 \mathrm{~mm}, \pm 5 \mathrm{~mm}, 0)$.

We define the magnetization of the superconductors, $M_{\mathbf{z}}$, as the vector potential of the current, $J_{c}=\nabla \times M_{z}$, which is a function of $x$ and $y$ only. Thus, $M_{\mathrm{z}}$ in each superconductor is described as in (1a) and (1b), and is shown diagrammatically in Fig. 2. The value of $J_{\mathrm{c}}$ is assumed to be $2 \times 10^{8} \mathrm{~A} / \mathrm{m}^{2}$, which is a typical representative value for high quality bulk high-temperature superconductors

$$
\begin{array}{ll}
M_{\mathrm{z}}=J_{\mathrm{c}}(r-a), & \text { when } r \leq a ; \\
M_{\mathrm{z}}=0, & \text { when } r>a,
\end{array}
$$




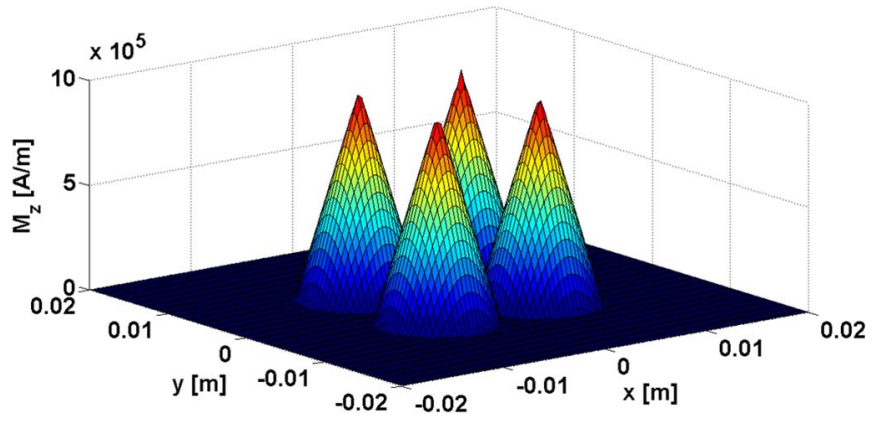

Fig. 2. The distribution of $M_{z}$ for the four-superconductor array.

where $a$ is the radius of each superconductor, $r$ is the distance between the observation point and the centre of any superconductor in the $x-y$ plane.

\section{Analytical Methods}

In this section, the analytical methods used in this analysis to solve the magnetic field of the superconductor array, shown in Fig. 1, are described, and a comparison is made between to determine their efficacy.

\section{A. Biot-Savart Method}

The Biot-Savart method has been used previously (see [9][11]) to analyse the field from an array of bulk superconductors. The magnetic field of a current loop can be calculated directly using the Biot-Savart law. For a superconductor with current density $J_{c}$, we can divide the superconductor into several concentric current loops at different heights [9]. The current flowing in each of these current loops is given by

$$
I=J_{\mathrm{c}} \Delta w \Delta t
$$

where $\Delta w$ and $\Delta t$ are the width and thickness, respectively, of each current loop.

To compare with existing literature, we assume $\Delta w=\Delta t=$ $0.1 \mathrm{~mm}$, based on [9]. The magnetic field distribution at $z=$ $6 \mathrm{~mm}$, i.e., $1 \mathrm{~mm}$ above the top of the superconductors, is calculated. The range of the area analysed is between $x=$ $\pm 10 \mathrm{~mm}$ and $y= \pm 10 \mathrm{~mm}$. The plane at $z=6 \mathrm{~mm}$ is divided into a grid of $101 \times 101$ elements and the field is calculated at all of these points. It is necessary to calculate only one quarter of the grid points due to symmetry of the model so, therefore, only $51 \times 51$ points are required. The field distribution at $z=6 \mathrm{~mm}$ is shown in Fig. 3. This result is similar to the result presented in [9] and the maximum $z$ component of flux density $B_{\mathrm{z}}$ is $0.300 \mathrm{~T}$ (cf. $0.297 \mathrm{~T}$ calculated in [9]).

The calculation of the $51 \times 51$ points takes about 40 minutes using a standard laptop computer. The accuracy of this method is chiefly dependent on the number of divisions of current loops in the superconductor along the width and height. The more divisions, the more accurate the calculation is, but the number of divisions grows, the calculation takes a longer time.

This method provides the field at a single point and so, if we want the field at various points, as is usually the case, this must be repeated for each point, which can be a slow process.

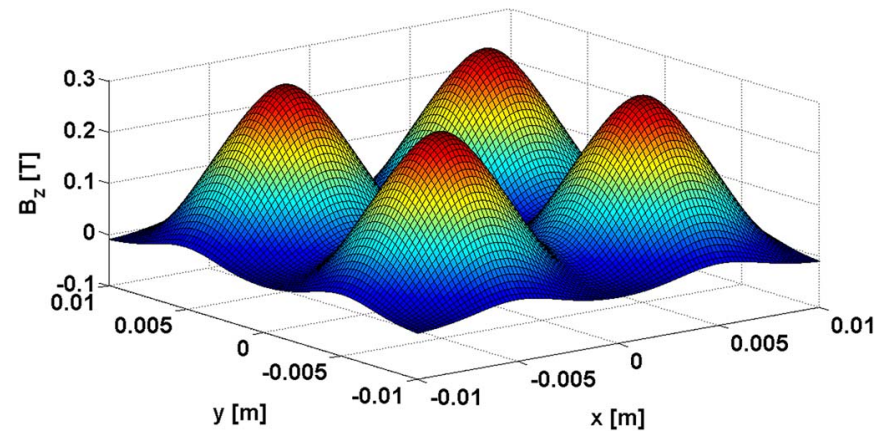

Fig. 3. The magnitude of the $z$ component of the magnetic flux density $B_{\mathrm{z}}$ in the plane at $z=6 \mathrm{~mm}, 1 \mathrm{~mm}$ above the bulk superconductor array, calculated using the Biot-Savart method.

\section{B. Fourier Transform Method}

The Fourier Transform (FT) method can convert a field distribution of any type into the sum of a harmonic series. The two-dimensional (2D) Fourier integral and inverse Fourier integral of the magnetization $M_{\mathrm{z}}$ is given by

$$
\begin{aligned}
M_{z k}\left(k_{x}, k_{y}\right) & =\int_{-\infty}^{\infty} \int_{-\infty}^{\infty} M_{z}(x, y) e^{-i\left(k_{x} x+k_{y} y\right)} d x d y \\
M_{z}(x, y) & =\frac{1}{4 \pi^{2}} \int_{-\infty}^{\infty} \int_{-\infty}^{\infty} M_{z k}\left(k_{x}, k_{y}\right) e^{i\left(k_{x} x+k_{y} y\right)} d k_{x} d k_{y}
\end{aligned}
$$

Equation (3a) and (3b) cannot be solved directly for circular samples, so a numerical solution needs to be found. First, $M_{\mathrm{z}}$ should be discretized at the grid points of the area to be calculated, and the area bounded by $x= \pm 20 \mathrm{~mm}$ and $y= \pm 20 \mathrm{~mm}$ is used here. Therefore, $M_{z}(x, y)$ becomes $M_{z}\left(x_{m}, y_{n}\right)$, where $\left(x_{m}, y_{n}\right)$ are the grid points

$$
\begin{aligned}
& x_{\mathrm{m}}=-2 d+(m-1) \Delta x, \quad m=1,2,3, \ldots, N \\
& y_{\mathrm{n}}=-2 d+(n-1) \Delta y, \quad n=1,2,3, \ldots, N,
\end{aligned}
$$

where $d$ is the diameter of the superconductors $(10 \mathrm{~mm}), N$ is the number of grid points, and $\Delta x=\Delta y=(4 d) /(N-1)$. Thus, the corresponding Fourier Transform becomes a Discrete Fourier Transform (DFT) with a discrete set of values. The frequencies for the DFT analysis are

$$
\begin{aligned}
& k_{x u}=\frac{2 \pi}{\Delta x} \frac{u-1}{N}, u=1,2,3, \ldots, \frac{N}{2}+1 \\
& k_{x u}=-\frac{2 \pi}{\Delta x} \frac{N-u+1}{N}, u=\frac{N}{2}+2, \frac{N}{2}+3, \frac{N}{2}+4, \ldots, N
\end{aligned}
$$

$$
\begin{aligned}
& k_{y v}=\frac{2 \pi}{\Delta y} \frac{v-1}{N}, v=1,2,3, \ldots, \frac{N}{2}+1 \\
& k_{y v}=-\frac{2 \pi}{\Delta y} \frac{N-v+1}{N}, v=\frac{N}{2}+2, \frac{N}{2}+3, \frac{N}{2}+4, \ldots, N .
\end{aligned}
$$


Thus, the integrals in (3a) and (3b) can be replaced by the following sums:

$$
\begin{aligned}
& M_{z k}\left(k_{x u}, k_{y v}\right) \\
& \quad=\sum_{m=1}^{N} \sum_{n=1}^{N} M_{z}\left(x_{m}, y_{n}\right) e^{-i\left(k_{x u}(m-1) \Delta x+k_{y v}(n-1) \Delta y\right)} \\
& M_{z}\left(x_{m}, y_{n}\right) \\
& \quad=\frac{1}{N^{2}} \sum_{u=1}^{N} \sum_{v=1}^{N} M_{z k}\left(k_{x u}, k_{y v}\right) e^{i\left(k_{x u}(m-1) \Delta x+k_{y v}(n-1) \Delta y\right)} .
\end{aligned}
$$

From the relationship between the magnetization $M_{\mathrm{z}}$ and the current density in the superconductors $J_{\mathbf{c}}, J_{c}=\nabla \times M_{z}$, we obtain

$$
\begin{aligned}
J_{x}\left(x_{m}, y_{n}\right)= & \frac{1}{N^{2}} \sum_{u=1}^{N} \sum_{v=1}^{N} i k_{y v} M_{z k}\left(k_{x u}, k_{y v}\right) \\
& \times e^{i\left(k_{x u}(m-1) \Delta x+k_{y v}(n-1) \Delta y\right)} \\
J_{y}\left(x_{m}, y_{n}\right)= & -\frac{1}{N^{2}} \sum_{u=1}^{N} \sum_{v=1}^{N} i k_{x u} M_{z k}\left(k_{x u}, k_{y v}\right) \\
& \times e^{i\left(k_{x u}(m-1) \Delta x+k_{y v}(n-1) \Delta y\right)} .
\end{aligned}
$$

The vector potential in the space $\boldsymbol{A}(x, y, z)$ can be obtained by solving Laplace's equation, $\nabla^{2} \boldsymbol{A}=0$. For a current density in the form of

$$
\begin{aligned}
& J_{x}(x, y)=\frac{1}{4 \pi^{2}} \int_{-\infty}^{\infty} \int_{-\infty}^{\infty} J_{x k}\left(k_{x}, k_{y}\right) e^{i\left(k_{x} x+k_{y} y\right)} d k_{x} d k_{y} \\
& J_{y}(x, y)=\frac{1}{4 \pi^{2}} \int_{-\infty}^{\infty} \int_{-\infty}^{\infty} J_{y k}\left(k_{x}, k_{y}\right) e^{i\left(k_{x} x+k_{y} y\right)} d k_{x} d k_{y}
\end{aligned}
$$

and considering the boundary conditions and superconductors of height $h$, the vector potential is

$$
\begin{aligned}
A_{x}(x, y, z)= & \frac{1}{4 \pi^{2}} \frac{\mu_{0}}{2} \int_{-\infty}^{\infty} \int_{-\infty}^{\infty} \frac{1}{k^{2}} J_{x k}\left(k_{x}, k_{y}\right) e^{i\left(k_{x} x+k_{y} y\right)} \\
& \times\left(e^{-k\left(z-\frac{h}{2}\right)}-e^{-k\left(z+\frac{h}{2}\right)}\right) d k_{x} d k_{y} \\
A_{y}(x, y, z)= & \frac{1}{4 \pi^{2}} \frac{\mu_{0}}{2} \int_{-\infty}^{\infty} \int_{-\infty}^{\infty} \frac{1}{k^{2}} J_{y k}\left(k_{x}, k_{y}\right) e^{i\left(k_{x} x+k_{y} y\right)} \\
& \times\left(e^{-k\left(z-\frac{h}{2}\right)}-e^{-k\left(z+\frac{h}{2}\right)}\right) d k_{x} d k_{y}
\end{aligned}
$$

where $k=\sqrt{k_{x}^{2}+k_{y}^{2}}$.

The relationship between the vector potential $\boldsymbol{A}(x, y, z)$ and the flux density $\boldsymbol{B}(x, y, z)$ is $\boldsymbol{B}=\nabla \times \boldsymbol{A}$, so the flux density $\boldsymbol{B}(x, y, z)$ can be obtained

$$
\begin{aligned}
& B_{x}(x, y, z) \\
& =\frac{1}{4 \pi^{2}} \frac{\mu_{0}}{2} \int_{-\infty}^{\infty} \int_{-\infty}^{\infty} \frac{1}{k} J_{y k}\left(k_{x}, k_{y}\right) e^{i\left(k_{x} x+k_{y} y\right)} \\
& \quad \times\left(e^{-k\left(z-\frac{h}{2}\right)}-e^{-k\left(z+\frac{h}{2}\right)}\right) d k_{x} d k_{y}
\end{aligned}
$$

$$
\begin{aligned}
& B_{y}(x, y, z) \\
& =-\frac{1}{4 \pi^{2}} \frac{\mu_{0}}{2} \int_{-\infty}^{\infty} \int_{-\infty}^{\infty} \frac{1}{k} J_{x k}\left(k_{x}, k_{y}\right) e^{i\left(k_{x} x+k_{y} y\right)} \\
& \quad \times\left(e^{-k\left(z-\frac{h}{2}\right)}-e^{-k\left(z+\frac{h}{2}\right)}\right) d k_{x} d k_{y} \\
& B_{z}(x, y, z) \\
& =\frac{1}{4 \pi^{2}} \frac{\mu_{0}}{2} \int_{-\infty}^{\infty} \int_{-\infty}^{\infty} \frac{1}{k^{2}}\left(i k_{x} J_{y k}\left(k_{x}, k_{y}\right)-i k_{y} J_{x k}\left(k_{x}, k_{y}\right)\right) \\
& \quad \times e^{i\left(k_{x} x+k_{y} y\right)}\left(e^{-k\left(z-\frac{h}{2}\right)}-e^{-k\left(z+\frac{h}{2}\right)}\right) d k_{x} d k_{y} .
\end{aligned}
$$

Therefore, for the current density described by (7a) and (7b), the flux density becomes

$$
\begin{aligned}
B_{x}( & \left.x_{m}, y_{n}, z\right) \\
= & -\frac{1}{N^{2}} \frac{\mu_{0}}{2} \sum_{u=1}^{N} \sum_{v=1}^{N} \frac{i k_{x u}}{k_{u v}} M_{z k}\left(k_{x u}, k_{y v}\right) \\
& \times e^{i\left(k_{x u}(m-1) \Delta x+k_{y v}(n-1) \Delta y\right)} \\
& \times\left(e^{-k_{u v}\left(z-\frac{h}{2}\right)}-e^{-k_{u v}\left(z+\frac{h}{2}\right)}\right) \\
B_{y}( & \left.x_{m}, y_{n}, z\right) \\
= & -\frac{1}{N^{2}} \frac{\mu_{0}}{2} \sum_{u=1}^{N} \sum_{v=1}^{N} \frac{i k_{y v}}{k_{u v}} M_{z k}\left(k_{x u}, k_{y v}\right) \\
& \times e^{i\left(k_{x u}(m-1) \Delta x+k_{y v}(n-1) \Delta y\right)} \\
& \times\left(e^{-k_{u v}\left(z-\frac{h}{2}\right)}-e^{-k_{u v}\left(z+\frac{h}{2}\right)}\right) \\
B_{z}( & \left.x_{m}, y_{n}, z\right) \\
= & \frac{1}{N^{2}} \frac{\mu_{0}}{2} \sum_{u=1}^{N} \sum_{v=1}^{N} M_{z k}\left(k_{x u}, k_{y v}\right) \\
& \times e^{i\left(k_{x u}(m-1) \Delta x+k_{y v}(n-1) \Delta y\right)} \\
& \times\left(e^{-k_{u v}\left(z-\frac{h}{2}\right)}-e^{-k_{u v}\left(z+\frac{h}{2}\right)}\right),
\end{aligned}
$$

where $k_{u v}=\sqrt{k_{x u}^{2}+k_{y v}^{2}}$. The coefficient when $k_{\mathrm{uv}}=0$ (or the Fourier integral of the flux density when $k_{\mathrm{xu}}=0, k_{\mathrm{yv}}=0$ ) should be zero according to the symmetric field distribution.

The magnetic field distribution at plane $z=6 \mathrm{~mm}$ can now be calculated. The number of grid points $N$ is 201 , so $\Delta x=\Delta y=(4 d) /(N-1)=(4 \times 10 \mathrm{~mm}) / 200=$ $0.2 \mathrm{~mm}$. The frequency resolution for this grid is $\Delta k_{\mathrm{x}}=$ $\Delta k_{\mathrm{y}}=(2 \pi) /(N \Delta x)=(2 \pi) /(201 \times 0.2 \mathrm{~mm}) \approx 156.298 \mathrm{~m}^{-1}$. Again, due to symmetry, only one quarter of the grid points are needed for the calculation, resulting in only $101 \times 101$ points. The field distribution in the plane at $z=$ $6 \mathrm{~mm}$ is shown in Fig. 4. The result is again similar to that shown in Fig. 3 and the result in [9], with the maximum $z$ component of flux density $B_{z}$ is $0.288 \mathrm{~T}$.

The calculation of these $101 \times 101$ points takes about one hour. As with the Biot-Savart method, the accuracy of the Fourier Transform method is mainly dependent on the size of the calculated area and the discretization of the grid used. 


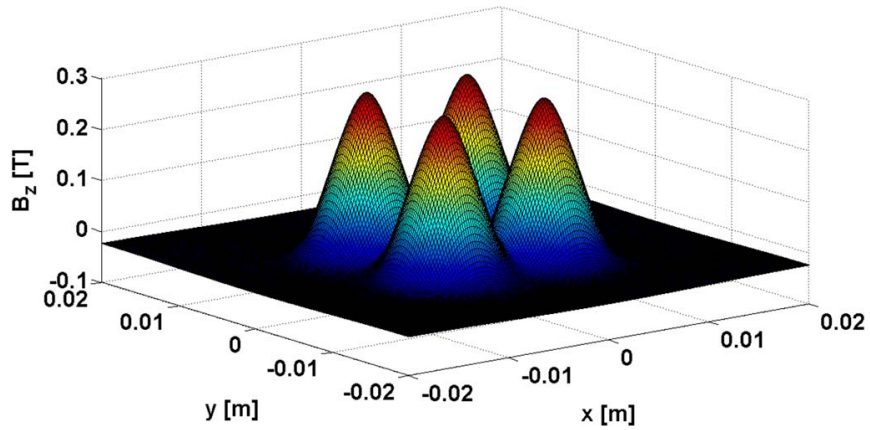

Fig. 4. The magnitude of the $z$ component of the magnetic flux density $B_{z}$ in the plane at $z=6 \mathrm{~mm}, 1 \mathrm{~mm}$ above the bulk superconductor array, calculated using the Fourier Transform method.

\section{Fast Fourier Transform Method}

The FT method can be accelerated using the Fast Fourier Transform (FFT) method. The principle is basically the same as above. However, we can simply use the 2D FFT function $f f t 2()$ and inverse FFT function if $f t 2()$ in Matlab to significantly improve the computational speed.

For a magnetization $M_{z}$, the sums in (6a) and (6b) can be implemented easily with the following commands in Matlab:

$$
\begin{aligned}
M_{\mathrm{z} k}\left(k_{\mathrm{xu}}, k_{\mathrm{yv}}\right) & =f f t 2\left(M_{\mathrm{z}}\left(x_{\mathrm{m}}, y_{\mathrm{n}}\right)\right) \\
M_{\mathrm{z}}\left(x_{\mathrm{m}}, y_{\mathrm{n}}\right) & =i f f t 2\left(M_{\mathrm{z} k}\left(k_{\mathrm{xu}}, k_{\mathrm{yv}}\right)\right) .
\end{aligned}
$$

For the current density $\boldsymbol{J}_{c}$ described earlier, and the relationship between $\boldsymbol{J}_{c}$ and $\boldsymbol{M}_{z}$ given in (7a) and (7b), the Fourier Transform of the current density $J_{\mathrm{c} k}\left(k_{\mathrm{xu}}, k_{\mathrm{yv}}\right)$ can be deduced from $M_{\mathrm{z} k}\left(k_{\mathrm{xu}}, k_{\mathrm{yv}}\right)$ in $(12 \mathrm{a})$

$$
\begin{aligned}
& J_{\mathrm{x} k}\left(k_{\mathrm{xu}}, k_{\mathrm{yv}}\right)=i k_{\mathrm{yv}} M_{\mathrm{z} k}\left(k_{\mathrm{xu}}, k_{\mathrm{yv}}\right) \\
& J_{\mathrm{y} k}\left(k_{\mathrm{xu}}, k_{\mathrm{yv}}\right)=-i k_{\mathrm{xu}} M_{\mathrm{z} k}\left(k_{\mathrm{xu}}, k_{\mathrm{yv}}\right) .
\end{aligned}
$$

With $J_{\mathrm{c} k}\left(k_{\mathrm{xu}}, k_{\mathrm{yv}}\right)$ calculated, the current density $J_{\mathrm{c}}\left(x_{\mathrm{m}}\right.$, $\left.y_{\mathrm{n}}\right)$ can be obtained easily using the if ft2() command.

From the relationship between the flux density and current density in (10a) and (10b), the Fourier Transform of the flux density $B_{k}\left(k_{\mathrm{xu}}, k_{\mathrm{yv}}\right)$ can be deduced from $J_{\mathrm{c} k}\left(k_{\mathrm{xu}}, k_{\mathrm{yv}}\right)$ in (13a) and (13b)

$$
\begin{aligned}
& B_{x k}\left(k_{x u}, k_{y v}\right) \\
& =\frac{\mu_{0}}{2} \frac{1}{k_{u v}} J_{y k}\left(k_{x u}, k_{y v}\right)\left(e^{-k_{u v}\left(z-\frac{h}{2}\right)}-e^{-k_{u v}\left(z+\frac{h}{2}\right)}\right) \\
& B_{y k}\left(k_{x u}, k_{y v}\right) \\
& =-\frac{\mu_{0}}{2} \frac{1}{k_{u v}} J_{x k}\left(k_{x u}, k_{y v}\right)\left(e^{-k_{u v}\left(z-\frac{h}{2}\right)}-e^{-k_{u v}\left(z+\frac{h}{2}\right)}\right)
\end{aligned}
$$

$$
\begin{aligned}
& B_{z k}\left(k_{x u}, k_{y v}\right) \\
& =\frac{\mu_{0}}{2} \frac{1}{k_{u v}^{2}}\left(i k_{x u} J_{y k}\left(k_{x u}, k_{y v}\right)-i k_{y v} J_{x k}\left(k_{x u}, k_{y v}\right)\right) \\
& \quad \times\left(e^{-k_{u v}\left(z-\frac{h}{2}\right)}-e^{-k_{u v}\left(z+\frac{h}{2}\right)}\right)
\end{aligned}
$$

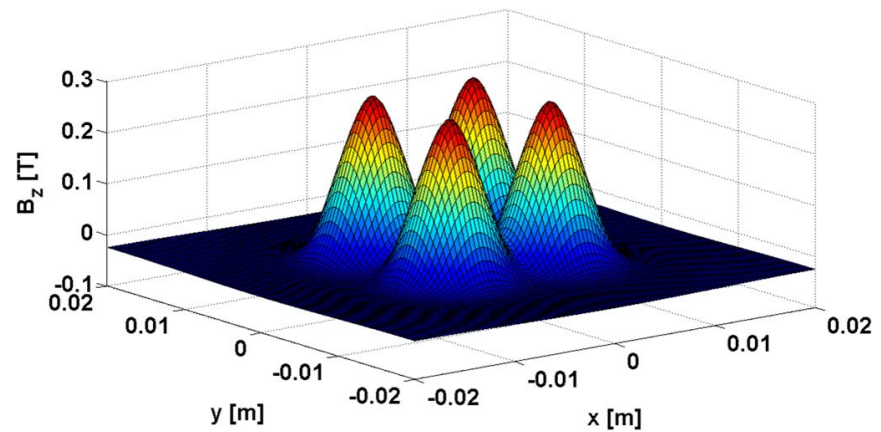

Fig. 5. The magnitude of the $\mathrm{z}$ component of the magnetic flux density $B_{z}$ in the plane at $\mathrm{z}=6 \mathrm{~mm}$, calculated using the Fast Fourier Transform method.

TABLE I

Comparison Between Different Methods in Different Terms

\begin{tabular}{ccccc}
\hline \hline Method & Speed & Memory & Accuracy & $\begin{array}{c}\text { Other } \\
\text { Comments }\end{array}$ \\
\hline $\begin{array}{c}\text { Biot- } \\
\text { Savart } \\
\text { (BS) }\end{array}$ & $\begin{array}{c}\text { Slow } \\
\text { (minutes) }\end{array}$ & Small & Accurate & $\begin{array}{c}\text { Solves the field } \\
\text { at an arbitrary } \\
\text { point }\end{array}$ \\
$\begin{array}{c}\text { Fourier } \\
\text { Transform } \\
\text { (FT) }\end{array}$ & $\begin{array}{c}\text { Slow } \\
\text { (hours) }\end{array}$ & Large & Varies & $\begin{array}{c}\text { Solves the field } \\
\text { for an area }\end{array}$ \\
$\begin{array}{c}\text { Fast } \\
\text { Fourier } \\
\text { Transform } \\
\text { (FFT) }\end{array}$ & $\begin{array}{c}\text { Fastest } \\
\text { (seconds) }\end{array}$ & Large & Accurate & $\begin{array}{c}\text { Solves the field } \\
\text { for an area }\end{array}$ \\
\hline
\end{tabular}

For $k_{\mathrm{xu}}=k_{\mathrm{yv}}=0, B_{\mathrm{xk}}(0,0)=0, B_{\mathrm{yk}}(0,0)=0, B_{\mathrm{zk}}(0$, $0)=0$. With $B_{\mathrm{k}}\left(k_{\mathrm{xu}}, k_{\mathrm{yv}}\right)$ calculated, the flux density $B\left(x_{\mathrm{m}}, y_{\mathrm{n}}, z\right)$ can be obtained easily by again using the if $f t 2()$ command.

The magnetic field distribution at plane $z=6 \mathrm{~mm}$ can now be calculated, and the calculated area is the same as for the FT method. The grid points $\left(x_{\mathrm{m}}, y_{\mathrm{n}}\right)$ and frequencies $\left(k_{\mathrm{xu}}, k_{\mathrm{yv}}\right)$ are listed in (4a) and (4b) and (5a) and (5b). The FFT method is fastest when the number of grid points is chosen to be $2^{\mathrm{n}}$ (where $\mathrm{n}$ is a positive integer). Here, the number of points $N=2048=2^{11}$ is chosen, making the size of the grid $\Delta x=\Delta y=(4 d) /(N-1)=$ $(4 \times 10 \mathrm{~mm}) / 2047 \approx 0.0195 \mathrm{~mm}$. The frequency resolution for this grid is $\Delta k_{\mathrm{x}}=\Delta k_{\mathrm{y}}=(2 \pi) /(N \Delta x)=(2 \pi) /(2048 \times$ $0.0195 \mathrm{~mm}) \approx 157.003 \mathrm{~m}^{-1}$. The field distribution in the plane at $z=6 \mathrm{~mm}$ is shown in Fig. 5, and is consistent with the other methods of calculation. The maximum $z$ component of the flux density $B_{\mathrm{z}}$ is $0.291 \mathrm{~T}$.

The calculation of the $2048 \times 2048$ points takes around 35 seconds, which makes it an extremely fast and accurate method. However, it does require a large amount of memory to store the data, which increases for finer grids/more accurate results. For example, for these $2048 \times 2048$ points, $577 \mathrm{Mb}$ of memory is required to store the computed data. Therefore, the FFT method can be an efficient method when the memory of the computer is big enough, which in this case is well within the limits of a standard laptop computer.

A summary of each of the methods carried out in this paper is provided in Table I using the metrics of speed, memory and accuracy to compare their performance. 


\section{CONCLUSION}

Detailed analysis of the magnetic field of arrays of bulk superconductors provides important information for the design and optimization of trapped flux-type superconducting electric machines, and can provide a useful reference tool for comparison with more complicated Finite Element Method-based solutions. In this paper, three different analytical methods-the Biot-Savart (BS) Method, the Fourier Transform Method (FT), and Fast Fourier Transform Method (FFT) - are used to calculate the magnetic field from an array of four fully magnetized bulk superconductors. The FFT method, while somewhat memory intensive, provides a fast and accurate method of solving such problems, in comparison to the BS and FT methods.

\section{ACKNOWLEDGMENT}

Dr. Mark Ainslie would like to acknowledge the support of a Royal Academy of Engineering Research Fellowship. Mr. Zejun Shen would like to acknowledge the support of the China Scholarship Council.

\section{REFERENCES}

[1] M. Tomita and M. Murakami, "High-temperature superconductor bulk magnets that can trap magnetic fields of over 17 tesla at $29 \mathrm{~K}, "$ Nature, vol. 421, no. 6922, pp. 517-520, Jan. 2002.

[2] J. H. Durrell et al., "A trapped field of $17.6 \mathrm{~T}$ in melt-processed bulk Gd-B-Cu-O reinforced with shrink-fit steel," Supercond. Sci. Technol., vol. 27, no. 8, Aug. 2014, Art. ID. 082001.

[3] S. Nariki, N. Sakai, and M. Murakami, "Melt-processed Gd-Ba-Cu-O superconductor with trapped field of $3 \mathrm{~T}$ at $77 \mathrm{~K}$," Supercond. Sci. Technol., vol. 18, no. 2, pp. 126-130, Feb. 2005.
[4] E. H. Ailam et al., "Design and testing of a superconducting rotating machine," IEEE Trans. Appl. Supercond., vol. 17, no. 1, pp. 27-33, Mar. 2007.

[5] J. R. Hull and M. Murakami, "Applications of bulk high-temperature superconductors," Proc. IEEE, vol. 92, no. 10, pp. 1705-1718, Oct. 2004.

[6] D. Zhou et al., "An overview of rotating machine systems with hightemperature bulk superconductors," Supercond. Sci. Technol., vol. 25 , no. 10 , Oct. 2012, Art. ID. 103001.

[7] M. D. Ainslie et al., "Numerical analysis and finite element modelling of an HTS synchronous motor," Phys. C, Supercond., vol. 470, no. 20, pp. 1752-1755, Nov. 2010.

[8] V. L. Chechurin and M. Sarma, "Computing models for the magnetic field calculation of superconducting electric machines with the help of scalar magnetic potential," IEEE Trans. Magn., vol. MAG-12, no. 6, pp. 10481049, Nov. 1976.

[9] A. Aydýner and E. Yanmaz, "Numerical calculation of trapped magnetic field for single and multiple bulk superconductors," J. Supercond. Nov. Magn., vol. 23, no. 4, pp. 457-463, May 2010.

[10] H. Fukai, M. Tomita, M. Murakami, and T. Nagatomo, "The effect of geometry on the trapped magnetic field in bulk superconductors," Supercond. Sci. Technol., vol. 15, no. 7, pp. 1054-1057, Jul. 2002.

[11] A. Aydýner and E. Yanmaz, "Numerical calculation of trapped magnetic field for square and cylindrical superconductors," Supercond. Sci. Technol., vol. 18, no. 7, pp. 1010-1015, Jul. 2005.

[12] T. Tokumasu, H. Matsumoto, K. Ito, and S. Oshima, "Magnetic field analysis of quick response type superconducting generator," IEEE Trans. Magn., vol. 30, no. 5, pp. 3713-3716, Sep. 1994.

[13] X. H. Jiang and A. M. Campbell, "Numerical calculation of magnetic fields in melt processed YBCO magnets," IEEE Trans. Appl. Supercond., vol. 7, no. 2, pp. 1213-1215, Jun. 1997.

[14] Y.-D. Chiin, Y.-H. Kim, J. Lee, J.-P. Hoiig, and J.-W. Lee, "Finite element analysis of magnetic field in high temperature bulk superconductor," IEEE Trans. Appl. Supercond., vol. 11, no. 1, pp. 2000-2003, Mar. 2001.

[15] M. D. Ainslie et al., "Modelling and comparison of trapped fields in (RE)BCO bulk superconductors for activation using pulsed field magnetization," Supercond. Sci. Technol., vol. 27, no. 6, Apr. 2014, Art. ID. 065008 . 